\title{
Role of primary cilium in pancreatic ductal adenocarcinoma (Review)
}

\author{
BERINA SABANOVIC, MATTEO GIULIETTI and FRANCESCO PIVA \\ Department of Specialistic Clinical and Odontostomatological Sciences, \\ Polytechnic University of Marche, I-60131 Ancona, Italy
}

Received May 21, 2020; Accepted August 21, 2020

DOI: $10.3892 /$ ijo.2020.5121

\begin{abstract}
The primary cilium is a non-motile cellular structure extending from the apical membrane of epithelial cells that is involved in several processes due to its ability to receive and elaborate different signals. Ciliogenesis and its obliteration are essential for proliferating cells, and several signalling pathways are responsible for their regulation. In fact, the primary cilium is a central hub for numerous signalling pathways implicated in a variety of biological processes, such as the Hedgehog, mammalian target of rapamycin and Wnt pathways. Loss of primary cilia has been recently correlated with different types of tumours, including pancreatic ductal adenocarcinoma (PDAC). K-Ras and HDAC2 were recently identified as possible ciliogenesis regulators in PDAC, likely acting through Aurora A kinase (AURKA) which, in turn, controls inositol polyphosphate-5-phosphatase E. However, the exact molecular mechanisms underlying this regulatory effect remain to be fully elucidated. In the present study, the regulation of the main genes involved in primary cilia assembly/resorption was reconstructed showing the links with the Hedgehog and phosphoinositide 3-kinase/AKT pathways. Finally, by analysing gene expression databases, the regulatory genes that have high probability to be associated with prognosis, histological grade and pathological stage in patients with PDAC have been highlighted. However, further experimental studies are required to reach definitive conclusions on the roles of these genes. Improving our understating of ciliogenesis and its regulators may help develop ciliotherapies using histone deacetylase and AURKA inhibitors, which may induce re-differentiation of tumour cells into normal cells by reducing tumour growth or inducing apoptosis of cancer cells.
\end{abstract}

Correspondence to: Dr Matteo Giulietti, Department of Specialistic Clinical and Odontostomatological Sciences, Polytechnic University of Marche, Via Brecce Bianche, I-60131 Ancona, Italy

E-mail:m.giulietti@univpm.it

Key words: cilia, pancreatic neoplasms, histone deacetylase 6, histone deacetylase 2 , aurora kinase

\section{Contents}

1. Structure and function of primary cilia

2. Assembly and disassembly of primary cilia: IFT and cell cycle

3. Primary cilia as mediators of signalling pathways

4. Loss of primary cilia and tumour development

5. PDAC and cilium

6. Association between primary ciliary regulatory genes and PDAC

7. Therapeutic strategies targeting primary cilia in PDAC

8. Conclusion

\section{Structure and function of primary cilia}

The primary cilium is a feature of almost all cell types, most commonly of epithelial cells.Primary cilia are cellular non-motile structures, composed of two centrioles, that extend from the basal body in the apical membrane. The cytoskeleton of the cilium, also referred to as axoneme, is composed of 9 post-translationally acetylated microtubule doublets in a 9+0 arrangement, lacking the central microtubule pair as in motile cilia $(1,2)$. Although all types of cilia have the same microtubule-based structure, their roles differ. Indeed, motile cilia are responsible for processes including cerebrospinal fluid flow and mucus clearance, whereas non-motile primary cilia have evolved in sensory organelles. Primary cilia receive numerous extracellular signals, such as mechanical stimuli, light and low-molecular-weight molecules (1-3). In addition to its structural role as major microtubule organization centre in mammalian cells, the basal body is also responsible for protein trafficking through the cilium. This protein exchange is regulated at the ciliary base comprising Y-linker proteins (transition zone, Y-ankles and ciliary gate). These linkers protrude from the outer microtubule pairs present in the transition zone to the ciliary membrane. The majority of the proteins located in the ciliary membrane have yet to be investigated for their functions; however, it is known that the ciliary membrane encompasses several receptor proteins, ion channels, protein transporters and sensory proteins. A number of these proteins are mutated in ciliopathies, i.e., diseases caused by dysregulation of primary cilia structure and function, such as autosomal dominant polycystic kidney disease, nephronophthisis and Meckel-Gruber syndrome (1-3). 


\section{Assembly and disassembly of primary cilia: IFT and cell cycle}

Two pathways of ciliogenesis have been described by Sorokin, namely the extracellular and intracellular pathways (4). In the extracellular pathway, the mother centriole anchors to the plasma membrane upon nucleation of axonemal microtubules. As the cilium derived directly from the mitotic spindle, and is the first cilium to emerge from the cell, it is named the 'primary cilium'. This type of ciliogenesis has been observed in all cells, including those that later develop a ciliated border. On the other hand, in the intracellular pathway, the axoneme elongation originates from the cytoplasm after association of the mother centriole with ciliary vesicles derived from the Golgi apparatus. This type of ciliogenesis has been observed in epithelial cells with a ciliated border (4). The assembly/disassembly of the primary cilium and trafficking of intermediary molecules of signalling pathways are dependent upon intraflagellar transport (IFT). IFT proceeds in two directions: i) Anterograde transport from the cilium base to the tip, catalysed by the dynein $2 / 1 \mathrm{~b}$ protein; and ii) retrograde transport from the tip to the cilium base, catalysed by kinesin-2 family proteins (KIF3A, KIF3B and KIF17). Proteins are transported to the base of the cilium by several pathways. This transport step involves IFT20 protein, exocyst-related small Rab GTPases and the BBSome complex, formed by seven Bardet-Biedl syndrome (BBS) proteins (BBS1, BBS2, BBS4, BBS5, BBS7, BBS8 and BBS9) that are located in the basal body and are involved in trafficking cargo to the primary cilium $(5,6)$. Jonassen et al identified that mutations in IFT proteins in murine models resulted in polycystic kidney disease (7).

Furthermore, assembly and disassembly of cilia are correlated with cell cycle status. As the mother centriole functions in the mitotic apparatus, it must be released from the plasma membrane and the primary cilia must disassemble prior to mitosis. In most cell types, the cilia are disassembled in the $M$ phase, re-assembled upon entry in the G1 phase, and they continue to grow in the G0 phase (8). However, Tucker et al have also observed ciliary resorption prior to the $\mathrm{S}$ phase in cells emerging from the quiescent state (9). Hence, ciliogenesis and its obliteration are crucial for proliferating cells and several signalling pathways are responsible for their regulation. The resorption of primary cilia has been well studied in human RPE1 (telomerase reverse transcriptase-immortalized retinal pigment epithelial) cell cultures. It has been demonstrated that growth factor-stimulated cell cultures, previously deprived of serum, caused ciliary disassembly through the activation of human enhancer of filamentation 1 (HEF1) and Aurora A kinase (AURKA), which sequentially activate histone deacetylase (HDAC)6 in the axoneme. Through deacetylation of axonemal tubules, HDAC6 promotes ciliary resorption (6). The phosphorylated KIF2A, a member of the kinesin-13 protein family, exhibits microtubule depolymerizing activity at the mother centriole, causing disassembly of the primary cilium coupled to the cell cycle (6). However, other proteins involved in this process have recently emerged, including the Pitchfork (PIFO) and dynein light chain Tctex-type 1 proteins. The PIFO protein is required specifically to control cilia retraction, as well as the liberation and duplication of the basal body/centrosome. It may act by stimulating AURKA activity at the basal body in a cell cycle-dependent manner. Cytoplasmic dynein 1 acts as a motor for the intracellular retrograde motility of vesicles and organelles along the microtubules $(6,8,10)$.

The primary cilia exhibit an interesting ability to adjust their morphology and size to external stimuli. The cilium can enlarge itself to improve its signal recognition capacity and, vice versa, the resorption of cilia results in reduced responsiveness to signals. The key mediators in these processes are second messengers and IFT. In cell types with apicobasal polarity, primary cilia are always localized to the apical surface. This is very important, since the apical membrane is directed towards the lumen of ducts; therefore, cilia are exposed to various chemical and mechanical signals (3).

Ciliogenesis appears to be autonomously regulated within cilium, since IFT is regulated through ciliary cAMP concentration in response to external signals, but also stimuli derived from the cell cycle may cause irreversible transition, such as assembly/disassembly of cilia at the entry and exit from the quiescent state $(3,11)$. Based on the experimental evidence obtained to date, it appears that ciliary resorption occurs in two waves, i) the G1 resorption, regulated mainly by AURKA-HDAC6 and polo-like kinase 1 (PLK1)-KIF2A loop; and ii) G2/M resorption, driven by NIMA-related kinase 2 (NEK2)-KIF24 (6). The NEK2 protein is a S/G2 kinase, which localizes to the distal portion of the mother centriole and is essential for correct cilium resorption at the G2/M transition (12). The substrate for this kinase is KIF24, a kinesin-13 family protein, the phosphorylation of which stimulates its microtubule-depolymerizing activity and prevents the extension of cilia in proliferating cells (13). As shown in Fig. 1, the AURKA-HDAC6 and NEK2-KIF24 complexes play distinct, consecutive roles during cilia resorption as cells re-enter the cell cycle from the quiescent state (6).

\section{Primary cilia as mediators of signalling pathways}

The primary cilium serves as a central hub for numerous signalling pathways implicated in a variety of biological processes, also known to be dysregulated in pancreatic ductal adenocarcinoma (PDAC), including the Hedgehog, Wnt and mammalian target of rapamycin (mTOR) pathways, which have been extensively investigated $(1,2,5)$. Since the primary cilium acts like a cellular antenna for several ligands, it is enriched of all the components, both receptors and effectors, of the Hedgehog, Wnt and mTOR signalling pathways. Moreover, the IFT motor proteins allow the trafficking of these components within the cilium and, therefore, they can modulate the activity of these signalling pathways $(1,2,5)$.

In numerous tumours characterized by Hedgehog dysregulation, it is reported that tumour cells lack primary cilia in comparison with normal neighbouring cells $(14,15)$. The consequence of the absence of the primary cilium is context-dependent, as it may lead to the induction or the suppression of Hedgehog pathway-dependent tumorigenesis $(1,2,5)$. The role of primary cilia in canonical Wnt signalling remains a subject of discussion, but it has been acknowledged that normal ciliogenesis is fundamental for the non-canonical Wnt/planar cell polarity pathway. Studies on the mTOR pathway in renal cells have proposed a model of 

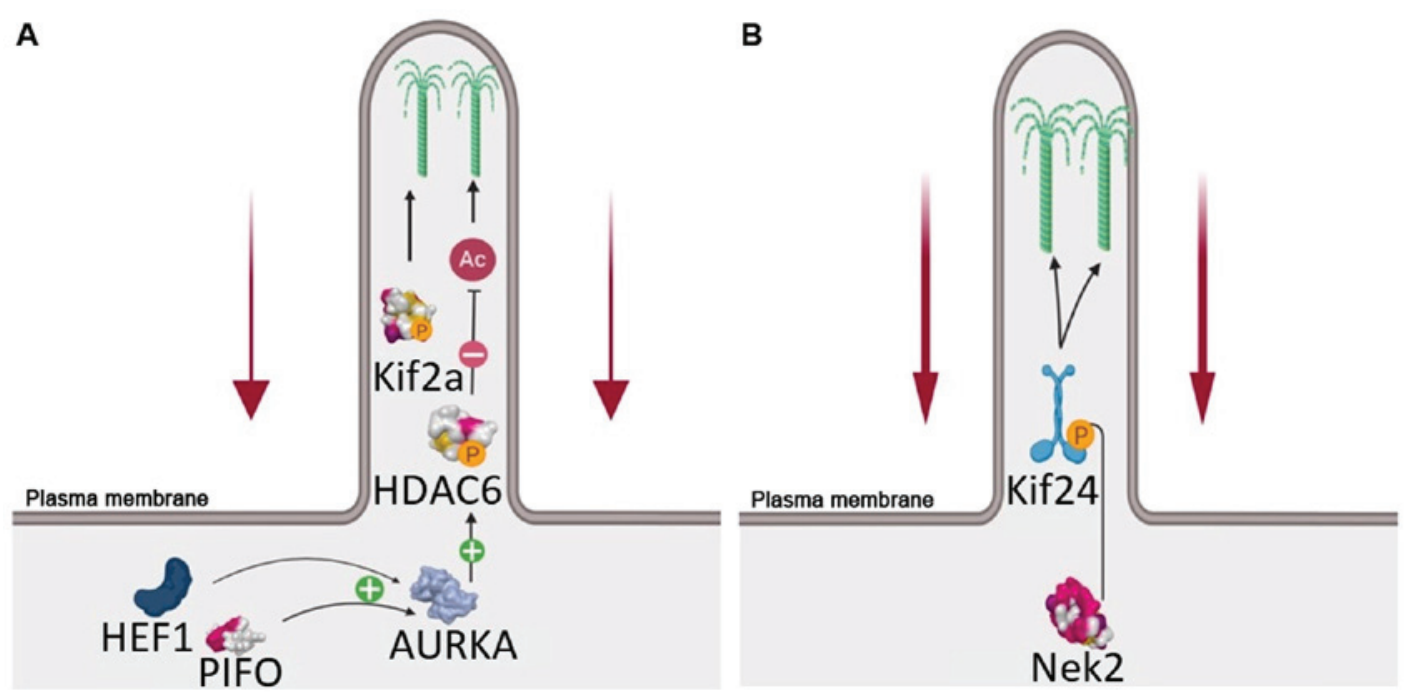

Figure 1. The primary cilium. The ciliary resorption is regulated by two protein loops: (A) AURKA-HDAC6 in the G1 phase of cell cycle and (B) NEK2-KIF24 in the G2/M phase of cell cycle. AURKA, Aurora A kinase; HDAC6, histone deacetylase 6; NEK2, NIMA-related kinase 2; KIF, kinesin family member.

cilium-dependent mTOR signalling. In particular, it has been reported that flow shear stress causes cilium bending, which consequently inhibits mTOR complex 1 (mTORC1) $(2,5)$. Upon cilium bending caused by fluid flux, the Leishmania protein kinase is transported to the basal body where another kinase, AMP-activated protein kinase, is phosphorylated, which, in turn, leads to the activation of the tuberous sclerosis (TSC)1-TSC2 complex, Rheb and, finally, the inhibition of mTORC1 (2).

\section{Loss of primary cilia and tumour development}

Various genomic and proteomic studies have identified $\sim 1,600$ genes essential for ciliary structure and function in mammals (16). Loss of primary cilia has been observed in various tumours, suggesting its involvement in carcinogenesis through aberrant signalling pathways. Development of tumours characterized by primary cilia absence is probably due to alterations in signalling pathways, which likely lead cells into a hyperproliferative state and promote migration and invasion (17).

Some initial results have already been proposed; for example, in cholangiocarcinoma (CCA), ciliary expression was found to be decreased both in human samples and human cell lines (17). It has been proposed that HDAC6 plays an important role in the regulation of ciliogenesis in CCA. It is believed that CCA and PDAC cells share a common mechanism of cilia loss, since K-Ras is responsible for HDAC6 activation $(8,15,18)$. Other tumours, such as prostate, colorectal, breast and melanoma, similarly display loss of primary cilia, but the underlying mechanisms remain to be elucidated (19-22). The reduction in the number of primary cilia has been also observed in ovarian cancer, renal cancer and glioblastoma. The underlying mechanism causing reduction of cilia in ovarian cancer is associated with overexpression and constant localization of AURKA to the ciliary basal body (23). HDAC6 plays an important role in the reduction of cilia number in renal cell carcinoma; in particular, it is activated as a consequence of an interaction among AURKA, $\beta$-catenin and HEF1 upon binding of von Hippel-Lindau to the microtubules (24).

\section{PDAC and cilium}

The pancreatic acinarcells undergo metaplastic acinar-to-ductal metaplasia, which lead to pancreatic intraepithelial neoplasia (PanIN), which may develop into PDAC. The majority of the PDAC cases harbour K-Ras gene mutations. Furthermore, the majority of human PanIN and PDAC lesions are devoid of primary cilia (25).

The study of Quilichini et al addressed the function of pancreatic ducts and primary cilia in postnatal pancreatic tissue homeostasis (26). The authors focused on hepatocyte nuclear factor (Hnf) $1 \mathrm{~b}$, a transcription factor that controls several genes important for pancreatic duct morphogenesis during development. Pancreatic cells with mutated Hnflb exhibit a major decrease in expression of genes involved in cystic disease, the ductal cells do not have primary cilia and have a 1.4-fold higher proliferation rate compared with control cells. The effects of Hnflb inactivation (loss of primary cilia, duct proliferation and dilatation) generate fibrosis, inflammatory infiltration, lipomatosis and activation of epithelial-to-mesenchymal transition, consequently leading to chronic pancreatitis and PanINs. Furthermore, a significant decrease in the expression of polycystic kidney and hepatic disease 1 (fibrocystin), cystin 1 , secreted phosphoprotein 1, prospero homeobox 1 and IFT88 (Tg737/IFT88) was observed. These genes are involved in maintenance of the ciliary and/or tubular architecture (26).

It has been demonstrated that PDAC cells lack primary cilia, independently of their proliferation status (15), by studies that have used human tissue specimens, the PANC-1 cell line and murine models. Instead, it was more recently demonstrated that primary cilia in the epithelium are gradually lost during pancreatic carcinogenesis, and this loss is accompanied by gain of primary cilia in the surrounding stroma (Table I). Moreover, the BxPc3, Capan1, MiaPaCa2, and PANC-1 PDAC cell lines do not lose primary cilia under conditions of nutrient or oxygen deprivation (27). 
Table I. Fraction of pancreatic cells with primary cilia.

Type of cell

Type of tissue

Normal pancreas

Chronic pancreatitis

Pancreatic intraepithelial

neoplasia

$1 \mathrm{~A}$

$1 \mathrm{~B}$

2

3

Pancreatic ductal adenocarcinoma

(no. of samples)

7

4

2

Intraductal papillary-mucinous

neoplasia (no. of samples)

\begin{tabular}{lll} 
Benign (4) & 0.0 & 8.3 \\
Malignant (8) & 0.0 & 8.3 \\
\hline
\end{tabular}

At the molecular level, these studies demonstrated that, by inhibiting the downstream effectors of the K-Ras pathway, mitogen-activated protein kinase kinase (MEK) and phosphoinositide 3-kinase (PI3K), ciliogenesis was fully restored, suggesting that K-Ras may be involved in the regulation of ciliogenesis in PDAC $(15,28)$. Although some studies have previously reported that AURKA drives primary cilia resorption by activation of HDAC6, this does not appear to be the case in PDAC. Indeed, Kobayashi et al, in an attempt to explain how loss of primary cilia affects PDAC development, revealed that HDAC6 is not involved in ciliation of PANC-1 cells. Depletion experiments with siRNA molecules suggested that HDAC2 is responsible for inhibition of primary ciliogenesis. Similar results have been obtained from other PDAC cell lines, such as CFPAC1 and KrasPDEC, suggesting that HDAC2 inhibition is a common characteristic of PDAC cells (28). HDAC2 is known to be overexpressed in PDAC lesions in comparison with normal duct epithelial cells. Furthermore, AURKA was also identified as a downstream target of K-Ras and HDAC2 in the suppression of ciliogenesis. It is possible that HDAC2 positively controls transcription of AURKA, which is overexpressed or amplified in several types of cancer, including PDAC (29). Moreover, HDAC6 phosphorylated by AURKA is involved in ciliary axoneme microtubule deacetylation, leading to disassembly of primary cilia in non-tumour cells. However, in PDAC models, inhibition of HDAC6 was not efficient against ciliation and treatment of PANC-1 cells with AURKA inhibitors led to a significant restoration of primary cilia (28). However, the role of primary cilia in PDAC development remains elusive.

AURKA regulates cell cycle entry and progression to the $\mathrm{M}$ phase, as well as ciliary resorption. Additionally, this protein kinase is involved in the mechanism of axonemal disassembly during cell exit from the G0 phase. When associated with other proteins, namely HEF1 (NEDD9) and Pitchfork (PIFO), AURKA exhibits increased catalytic activity and induces HDAC6 phosphorylation. This reaction may cause ciliary destabilization and resorption. HEF1 activation of AURKA has been observed after 1-2 $\mathrm{h}$, and after 18-24 h upon treatment with serum, suggesting that the HEF1-AURKA-HDAC6 loop is a key player in ciliary disassembly, even in the first phase of resorption (G1 resorption). However, this phenomenon has yet to be fully elucidated. The role of HDAC6 in ciliogenesis has yet to be clearly determined due to the opposite results of studies on mice with HDAC6 mutations who do not present ciliopathies (8). Furthermore, other AURKA regulators have been identified, such as calmodulin and $\mathrm{Ca}^{2+}$. Inositol polyphosphate-5-phosphatase E (INPP5E), a ciliary lipid phosphatase, removes the 5-phosphate group from $\mathrm{PI}(3,4,5) 3 \mathrm{P}$ and $\mathrm{PI}(4,5)$ $\mathrm{P} 2$, and has been correlated to AURKA activity for the stability of primary cilia. Furthermore, it has been recognized that INPP5E maintains PI $(4,5) \mathrm{P} 2$ at low or minimum levels in order to ensure correct trafficking of Hedgehog proteins and, hence, it is implicated in Hedgehog signalling regulation in primary cilia (30-32). The control of the disassembly and resorption of primary cilia is shown in Fig. 2. In particular, AURKA can phosphorylate INPP5E, subsequently increasing its 5-phosphatase activity, and its highest affinity is for $\mathrm{PI}(3,4,5) \mathrm{P} 3$ (31-33). INPP5E interacts with the $\mathrm{p} 85$ subunit of PI3K, leading to the inhibition of the PI3K/AKT signalling pathway (34). In fact, 293 cells stably transfected with the INPP5E gene were found to be highly susceptible to FAS-induced apoptosis and cell growth was suppressed (35). Accumulation of $\mathrm{PI}(4,5) \mathrm{P} 2$ in the distal half of the cilium led to decapitation by controlling intraciliary F-actin assembly, followed by resorption of the primary cilium (36). PI(4,5)P2 accumulation caused by INPP5E mutations negatively regulates the Sonic Hedgehog (SHH) pathway $(32,37,38)$. However, in order to cause ciliary resorption, it is necessary that AURKA phosphorylates KIF2A and HDAC6 (or HDAC2 in PDAC), but not INPP5E.

Seeley et al studied the abundance and distribution of primary cilia in 17 patients with PDAC to establish whether primary ciliogenesis is disrupted in cancer (15). The abundance of primary cilia in histologically normal tissues (negative control samples from each patient) generally varied. On the other hand, almost all pancreatic cancer samples were devoid of primary cilia, independently of tumour stage, suggesting that absence of primary cilia is a conserved characteristic of tumour cells. Authors have reported lack of primary cilia in PanIN-1, 2 and 3 lesions; therefore, the arrest in ciliary formation would occur in the earliest stages of PDAC development. Since centro-acinar and ductal pancreatic cells are ciliated, PanIN formation would involve ciliogenesis arrest of either cell type. Based on the results, the authors concluded that i) lack of primary cilia is independent of proliferation status, ii) arrest of ciliary formation is reversible and iii) overactivation of the K-Ras pathway arrests ciliogenesis in PDAC (15). Primary cilia have been restored in PDAC upon blocking K-Ras signalling with PI3K and MEK inhibitors. This result suggested that activated K-Ras signalling is directly involved in the inhibition of ciliogenesis. Since overstimulation of the Hedgehog pathway is a hallmark of PDAC, and absence of 


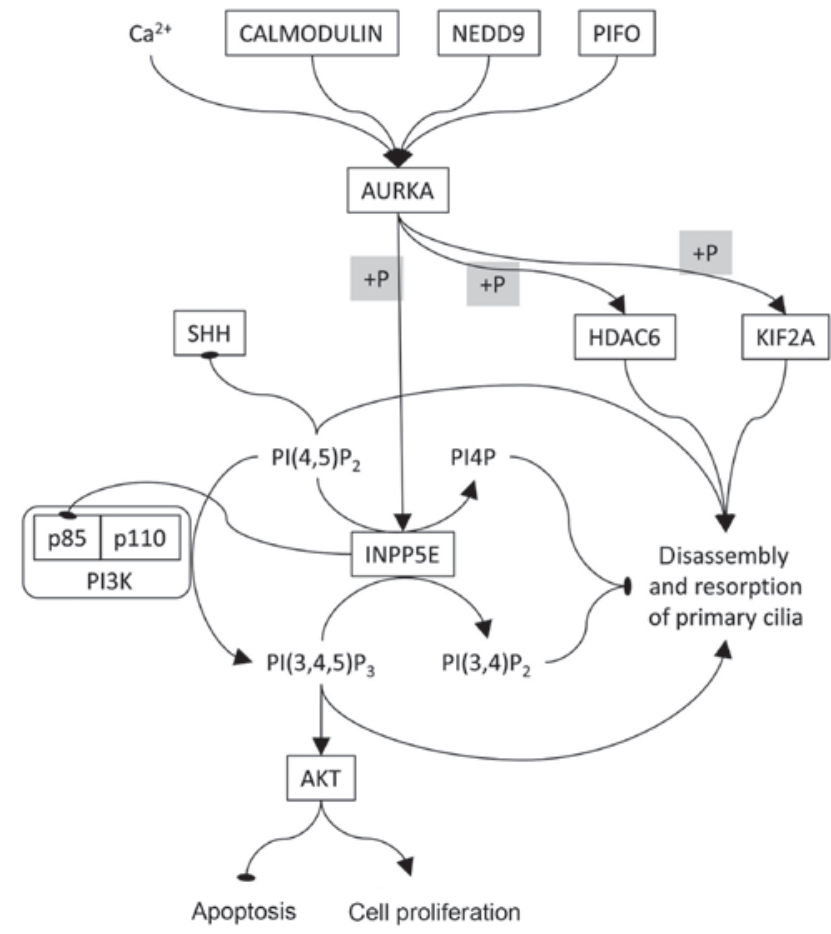

Figure 2. AURKA is the key element controlling the disassembly and resorption of the primary cilium. AURKA is controlled by calcium and various proteins, including calmodulin, NEDD and PIFO. AURKA activates INPP5E by phosphorylation. INPP5E converts PI(4,5)P2 to PI4P and $\mathrm{PI}(3,4,5) \mathrm{P} 3$ to $\mathrm{PI}(3,4) \mathrm{P} 2$. $\mathrm{PI} 3 \mathrm{~K}$ converts $\mathrm{PI}(4,5) \mathrm{P} 2$ to $\mathrm{PI}(3,4,5) \mathrm{P} 3$ which, in turn, activates AKT, leading to resistance to apoptosis and cell proliferation. In order to allow disassembly and resorption of the primary cilium, AURKA must negatively regulate INPP5E. Oval-ending connectors indicate inhibition. AURKA, Aurora A kinase; PIFO, Pitchfork; NEDD, neural precursor cell expressed developmentally downregulated protein; PI, phosphatidylinositol; INPP5E, inositol polyphosphate-5-phosphatase E; HDAC6, histone deacetylase 6; KIF, kinesin family member; SHH, Sonic Hedgehog.

primary cilia upregulates this pathway, it has been hypothesized that deciliation is involved in tumour progression via upregulation of the Hedgehog pathway (25).

In the study of Emoto et al, primary cilia were identified in 100 PDAC patients who received no therapy prior to initial surgery (39). Among the cases presenting primary cilia (25 out of 100), almost all (24 out of 25) had lymph node metastasis, and this frequency was higher than in cases where primary cilia were absent. There was a statistically significant association between overall survival (OS) and the tumour size, grade, lymph node metastasis and presence of primary cilia. In particular, patients with cancer with primary cilia had a poorer outcome compared with patients without primary cilia (39). It has been reported that SHH is highly expressed in PDAC tissues, in PanIN lesions and in several pancreatic cancer cells deriving from primary tumours and metastases, suggesting that the Hedgehog pathway plays a key role in the early and late phases of PDAC tumorigenesis (40). Similarly, Emoto et al demonstrated high levels of SHH expression in the PANC-1 and Aspc-1 pancreatic cancer cell lines (39). SHH can activate stromal cells, abundant in primary cilia, which may consequently stimulate epithelial cell proliferation and, therefore, tumorigenesis (39). By overexpressing SHH in mouse models, it has been demonstrated that pancreatic primary tumours were larger and the rates of tumour invasion and metastasis to the liver, spleen and peritoneum were higher (41). In addition, primary cilia and the Hedgehog receptor SMO were co-present in stromal cells, both in primary tumours and metastases, but were absent in primary tumour cells and metastatic cells. Instead, SHH production follows an opposite pattern. Moreover, $\mathrm{SHH}$ enhances the paracrine expression of matrix metalloproteinase 9. These results suggest that $\mathrm{SHH}$ acts on stromal cells, enriched in primary cilia, in order to regulate the tumour microenvironment and promote metastasis formation (41).

Deng et al investigated the biological functions of cilia during the malignant transformation by knocking down Tg737/IFT88 and KIF3A (a subunit of the kinesin-II complex), two crucial components of cilia, in mouse embryonic fibroblasts and HPDE6C7 (an immortalized epithelial cell line derived from normal human pancreatic duct epithelial cells) (42). This study demonstrated that the mevalonate (MVA) pathway is activated upon inactivation of ciliogenesis and may accelerate the oncogene-induced transformation of normal cells both in vitro and in vivo. Another important characteristic of the MVA pathway products are many non-sterol isoprenoids, such as farnesyl pyrophosphate and geranylgeranyl diphosphate. These two products are known mediators of protein prenylation, and their substrates also include small $\mathrm{G}$ proteins such as Ras, Rho and Rac, which are implicated in a number of tumours, including PDAC (42).

\section{Association between primary ciliary regulatory genes and PDAC}

The Xena Functional Genomics Explorer tool (https://xenabrowser.net/) was used to explore the associations between RNAseq expression of key genes in primary cilium regulation and phenotypic variables in the pancreatic adenocarcinoma dataset from The Cancer Genome Atlas of 178 patients. As shown in Table II, by analysing the Kaplan-Meier curves and using log-rank tests, it was revealed that the expression of the AURKA, PLK1, NEK2, HDAC6 and INPP5E genes was associated with OS and progression-free survival (PFS). In particular, lower expression of AURKA, PLK1 and NEK2, and higher expression of HDAC6 and INPP5E, were associated with better prognosis. Instead, higher expression of PIFO was only associated with better OS. The gene expression of AURKA, PLK1, NEK2, KIF2A, NEDD9, calmodulin (CALM)1, CALM2 and CALM3 is statistically significant higher in tumours compared with in normal tissues (data derived from the Genotype-Tissue Expression database; www.gtexportal.org). On the other hand, the expression of PIFO, HDAC6 and INPP5E is lower in tumours compared with that in normal tissues. By using one-way ANOVA, it was demonstrated that the gene expression of AURKA, PLK1 and NEK2 increases proportionally to the tumour grade, whereas CALM1 displays an inverse proportionality. Finally, no gene expression was found to be associated with pathological stage. These expression data indicate that AURKA induces ciliary resorption, it is a negative prognostic index, and it is more highly expressed in tumours of higher histological grade. As regards INPP5E, which blocks ciliary resorption, the data suggest that its higher expression improves the prognosis. Of note, high PIFO expression, which induces ciliary resorption, 
Table II. Survival and expression analyses of primary cilium-related genes in pancreatic ductal adenocarcinoma.

\begin{tabular}{|c|c|c|c|c|c|}
\hline \multirow[b]{2}{*}{ Genes } & \multicolumn{2}{|c|}{ Survival } & \multicolumn{3}{|c|}{ Expression } \\
\hline & $\begin{array}{l}\text { Association } \\
\text { with OS } \\
\text { (P-value) }\end{array}$ & $\begin{array}{l}\text { Association } \\
\text { with PFS } \\
\text { (P-value) }\end{array}$ & $\begin{array}{c}\text { Expression in } \\
\text { primary tumor } \\
\text { vs. normal tissue } \\
\text { (P-value) }\end{array}$ & $\begin{array}{l}\text { Association } \\
\text { with histological } \\
\text { tumor grade } \\
\text { (P-value) }\end{array}$ & $\begin{array}{c}\text { Association with } \\
\text { pathological stage } \\
\text { (P-value) }\end{array}$ \\
\hline AURKA & Inverse $(0.01774)$ & Inverse (0.02812) & Higher (4.995e-83) & Direct $(0.001278)$ & NS \\
\hline PLK1 & Inverse $(0.007640)$ & Inverse $(0.005791)$ & Higher (4.142e-71) & Direct (0.000005639) & NS \\
\hline NEK2 & Inverse $(0.003376)$ & Inverse (0.01698) & Higher (3.723e-104) & Direct (0.000008372) & NS \\
\hline KIF2A & NS & NS & Higher (5.708e-8) & NS & NS \\
\hline NEDD9 & NS & NS & Higher (3.901e-38) & NS & NS \\
\hline CALM1 & NS & NS & Higher (3.047e-12) & Inverse $(0.000006607)$ & NS \\
\hline CALM2 & NS & NS & Higher (2.185e-31) & NS & NS \\
\hline CALM3 & NS & NS & Higher (6.951e-11) & NS & NS \\
\hline PIFO & Direct (0.03329) & NS & Lower (2.528e-21) & NS & NS \\
\hline HDAC6 & Direct (0.005232) & Direct $(0.0009176)$ & Lower (0.00002954) & NS & NS \\
\hline INPP5E & Direct $(0.0006044)$ & $\operatorname{Direct}(0.01807)$ & Lower (4.150e-13) & NS & NS \\
\hline
\end{tabular}

Log-rank test for Kaplan-Meier curves was used for OS and PFS. One-way ANOVA was used for stage- and grade-specific expression analysis. Welch's t-test was used for differential RNA-seq expression analysis between primary tumor patients and healthy subjects. Among tumor samples, only primary tumors have been considered $(\mathrm{n}=178)$. Expression data for 167 normal subjects were obtained from the Genotype-Tissue Expression portal. AURKA, Aurora A kinase; PLK1, polo-like kinase 1; NEK2, NIMA-related kinase 2; KIF, kinesin family member; NEDD, neural precursor cell expressed developmentally downregulated protein; CALM, calmodulin; PIFO, Pitchfork; HDAC6, histone deacetylase 6; INPP5E, inositol polyphosphate-5-phosphatase E; NS, not significant.

would be expected to worsen the prognosis; instead, our data demonstrated an improvement of prognosis.

\section{Therapeutic strategies targeting primary cilia in PDAC}

Currently, it is widely hypothesized that the therapies targeted at ciliary restoration, collectively termed ciliotherapies, may induce re-differentiation of tumour cells to normal cells, reduce tumour growth, or even initiate apoptosis of cancer cells. Of note, ciliotherapies would vary among different tumour types. However, it has been reported that HDAC6 activation is a common event among various tumours. Gradilone et al reported successful in vitro and in vivo results by targeting HDAC6 in CCA $(17,18)$. HDAC6 was targeted with shRNA or with the inhibitor tubastatin-A, resulting in restored primary cilia formation, decreased cell proliferation and anchorage-independent growth. Although some studies have previously reported that activation of HDAC6 drives primary cilia resorption, it does not appear to be the case in PDAC. Kobayashi et al treated PANC-1 cells with the HDAC6 inhibitor tubacin, or with HDAC6 siRNA, but no effect on primary cilia assembly was observed, although HDAC6 was efficiently inhibited (28). Since primary ciliogenesis in PANC-1 cells was induced by treatment with trichostatin A, a pan-HDAC inhibitor, further inhibitors (i.e., valproic acid, MS-275 and FK228/depsipeptide) and specific siRNAs have been used to determine the involvement of HDAC family members in cilia formation, and HDAC2 was found to be responsible for inhibition of primary ciliogenesis in PANC-1 cells and in other PDAC cell lines (28). HDAC2 likely suppresses primary cilia formation by controlling the expression of genes involved in cilia assembly and disassembly, including AURKA. Indeed, PANC-1 cells treated with AURKA inhibitors (alisertib and PHA-680632) exhibited marked restoration of primary cilia (28). Recently, in a 3D mouse pancreatic acinar cell culture that displays acinar-to-ductal metaplasia (a pancreatic pre-neoplastic lesion), the inhibition of AURKA by MLN8237 (alisertib) significantly increased ciliogenesis. Furthermore, tubacin-induced inhibition confirmed that HDAC6 is not involved in cilium disassembly in the pancreas (43).

Some clinical trials investigating the safety and efficacy of certain HDAC inhibitors in patients with PDAC are ongoing. The majority involves pan-HDAC inhibitors, such as vorinostat (ClinicalTrials.gov IDs NCT00667082, NCT00831493, NCT00983268, NCT02349867 and NCT00948688) and panobinostat (NCT01056601), or the class 1 inhibitors entinostat (NCT03925428, NCT03760614, NCT00020579 and NCT03250273) and romidepsin (NCT00379639 and NCT04257448) in combination with other chemotherapy drugs for PDAC. However, there are currently no ongoing clinical trials on HDAC2-specific inhibitors in PDAC patients. HDAC inhibitors, although they have shown promising results in preclinical models, are associated with certain difficulties in the clinical setting, likely due to their toxicity, which is associated with low specificity towards a single HDAC member (44). As regards AURKA, some clinical trials are focusing on its oral inhibitor, alisertib, in combination with gemcitabine or nab-paclitaxel (NCT01677559 and NCT01924260). However, due to the paucity of published preclinical and clinical results, 
the usefulness of HDAC or AURKA inhibitors in PDAC treatment remains to be clearly determined.

\section{Conclusion}

Primary cilia contain several proteins that act as receptors, ion channels and transporters; thus, they are able to receive various extracellular signals (mechanical stimuli, light, low-molecular-weight molecules, etc.). Assembly and disassembly of cilia are correlated with cell cycle status. In particular, most cell types undergo cilia disassembly in the $M$ phase and re-assembly upon entry in the G1 phase. Therefore, inhibition of ciliogenesis is crucial for proliferating cells, but its exact regulation mechanism remains unknown at present. Based on the evidence obtained to date, it appears that ciliary resorption is regulated by the AURKA-HDAC6 and PLK1-KIF2A loops in the G1 phase, and by the NEK2-KIF24 loop in the G2/M phase.

Lack of primary cilia has been reported in tumour cells characterized by dysregulation of certain signalling pathways. The consequences of ciliary absence may differ depending on the context; in some cases it may promote tumour growth, whereas in others it may act as an inhibitor. However, some of these pathways may work normally, even in the absence of cilia, although many ciliary proteins have been associated with signalling pathways. Therefore, it would be reasonable to suggest that signalling pathways may include cilium-dependent and -independent mechanisms. Loss of primary cilia has been observed in a number of tumour samples, indicating their role in carcinogenesis due to aberrant signalling, which may lead cells into a hyperproliferative state and increase their migration and invasion abilities. PDAC cells also lack primary cilia, independently of proliferation status. It has been suggested that K-Ras may be involved in ciliogenesis regulation in PDAC. Furthermore, HDAC2 may cause loss of primary cilia by regulating AURKA transcription independently of K-Ras.

In the present study, the gene regulation of AURKA and INPP5E was reconstructed and it was demonstrated that AURKA-mediated INPP5E phosphorylation blocks the resorption of primary cilia. On the other hand, the lack of phosphorylation of INPP5E activates the PI3K/AKT pathway, which promotes the proliferation and survival of cancer cells. Through analysing gene expression databases, it was highlighted which primary cilia regulatory genes have a high probability to be associated to prognosis, histological grade and pathological stage in patients with PDAC. As expected, a higher expression of AURKA (which induces primary cilia resorption) was found to be associated with lower OS and PFS and with higher histological degree. Similarly, the expression of INPP5E (which prevents primary cilia resorption) was associated with higher OS and PFS. However, further experimental studies are required to reach definitive conclusions on their roles. Indeed, the databases used herein store RNAseq data and not data on protein expression or enzymatic activity, which are yet to be evaluated.

It would be interesting to determine the precise downstream targets of AURKA phosphorylation, which may cause deciliation of pancreatic cells and PDAC progression, possibly via upregulation of the Hedgehog pathway. Many questions are to be answered regarding primary cilia and ciliogenesis regulation. A better understanding of this process would be useful for the development of therapies targeted at cilia restoration. These ciliotherapies may decrease tumour growth rate or stimulate apoptosis of cancer cells. However, although several clinical trials for HDAC and AURKA inhibitors are currently in progress, there is yet no validated clinical application for PDAC.

\section{Acknowledgements}

Not applicable.

\section{Funding}

No funding was received.

\section{Availability of data and material}

Not applicable.

\section{Authors' contributions}

BS and MG: writing and revision of the manuscript. BS, MG and FP: reference collection, discussion. FP: conception and design of the study. All authors have read and approved the final version of the manuscript.

\section{Ethics approval and consent to participate}

Not applicable.

\section{Patient consent for publication}

Not applicable.

\section{Competing interests}

The authors declare that they have no competing interests.

\section{References}

1. Higgins M, Obaidi I and McMorrow T: Primary cilia and their role in cancer. Oncol Lett 17: 3041-3047, 2019.

2. Pala R, Alomari N and Nauli SM: Primary Cilium-dependent signaling mechanisms. Int J Mol Sci 18: 2272, 2017.

3. Malicki JJ and Johnson CA: The Cilium: Cellular antenna and central processing unit. Trends Cell Biol 27: 126-140, 2017.

4. Sorokin SP: Reconstructions of centriole formation and ciliogenesis in mammalian lungs. J Cell Sci 3: 207-230, 1968.

5. Wheway G, Nazlamova L and Hancock JT: Signaling through the primary cilium. Front Cell Dev Biol 6: 8, 2018.

6. Izawa I, Goto H, Kasahara K and Inagaki M: Current topics of functional links between primary cilia and cell cycle. Cilia 4: 12, 2015

7. Jonassen JA, San Agustin J, Follit JA and Pazour GJ: Deletion of IFT20 in the mouse kidney causes misorientation of the mitotic spindle and cystic kidney disease. J Cell Biol 183: 377-384, 2008.

8. Pugacheva EN, Jablonski SA, Hartman TR, Henske EP and Golemis EA: HEF1-dependent Aurora A activation induces disassembly of the primary cilium. Cell 129: 1351-1363, 2007.

9. Tucker RW, Pardee AB and Fujiwara K: Centriole ciliation is related to quiescence and DNA synthesis in 3 T3 cells. Cell 17: 527-535, 1979.

10. Li A, Saito M, Chuang J, Tseng Y, Dedesma C, Tomizawa K, Kaitsuka T and Sung C: Ciliary transition zone activation of phosphorylated Tctex-1 controls ciliary resorption, S-phase entry and fate of neural progenitors. Nat Cell Biol 13: 402-411, 2011. 
11. Breslin L, Prosser SL, Cuffe S and Morrison CG: Ciliary abnormalities in senescent human fibroblasts impair proliferative capacity. Cell Cycle 13: 2773-2779, 2014.

12. Spalluto C, Wilson DI and Hearn T: Nek2 localises to the distal portion of the mother centriole/basal body and is required for timely cilium disassembly at the G2/M transition. Eur J Cell Biol 91: 675-686, 2012.

13. Kim S, Lee K, Choi J, Ringstad N and Dynlacht BD: Nek2 activation of Kif24 ensures cilium disassembly during the cell cycle. Nat Commun 6: 8087, 2015.

14. Moser JJ, Fritzler MJ and Rattner JB: Primary ciliogenesis defects are associated with human astrocytoma/glioblastoma cells. BMC Cancer 9: 448, 2009.

15. Seeley ES, Carrière C, Goetze T, Longnecker DS and Korc M: Pancreatic cancer and precursor pancreatic intraepithelial neoplasia lesions are devoid of primary cilia. Cancer Res 69: 422-430, 2009

16. Gherman A, Davis EE and Katsanis N: The ciliary proteome database: An integrated community resource for the genetic and functional dissection of cilia. Nat Genet 38: 961-962, 2006.

17. Gradilone SA, Pisarello MJL and LaRusso NF: Primary cilia in tumor biology: The primary cilium as a therapeutic target in cholangiocarcinoma. Curr Drug Targets 18: 958-963, 2017.

18. Gradilone SA, Radtke BN, Bogert PS, Huang BQ, Gajdos GB and LaRusso NF: HDAC6 inhibition restores ciliary expression and decreases tumor growth. Cancer Res 73: 2259-2270, 2013.

19. Kim J, Dabiri S and Seeley ES: Primary cilium depletion typifies cutaneous melanoma in situ and malignant melanoma. PLoS One 6: e27410, 2011.

20. Hassounah NB, Nagle R, Saboda K, Roe DJ, Dalkin BL and McDermott KM: Primary cilia are lost in preinvasive and invasive prostate cancer. PLoS One 8: e68521, 2013.

21. Yuan K, Frolova N, Xie Y, Wang D, Cook L, Kwon YJ, Steg AD Serra R and Frost AR: Primary cilia are decreased in breast cancer: Analysis of a collection of human breast cancer cell lines and tissues. J Histochem Cytochem 58: 857-870, 2010.

22. Ho L, Ali SA, Al-Jazrawe M, Kandel R, Wunder JS and Alman BA: Primary cilia attenuate hedgehog signalling in neoplastic chondrocytes. Oncogene 32: 5388-5396, 2013.

23. Egeberg DL, Lethan M, Manguso R, Schneider L, Awan A, Jørgensen TS, Byskov AG, Pedersen LB and Christensen ST: Primary cilia and aberrant cell signaling in epithelial ovarian cancer. Cilia 1: 15, 2012.

24. Dere R, Perkins AL, Bawa-Khalfe T, Jonasch D and Walker CL: $\beta$-catenin links von Hippel-Lindau to aurora kinase A and loss of primary cilia in renal cell carcinoma. J Am Soc Nephrol 26 553-564, 2015

25. Kobayashi T and Itoh $\mathrm{H}$ : Loss of a primary cilium in PDAC. Cell Cycle 16: 817-818, 2017

26. Quilichini E, Fabre M, Dirami T, Stedman A, De Vas M, Ozguc O, Pasek RC, Cereghini S, Morillon L, Guerra C, et al: Pancreatic ductal deletion of Hnflb disrupts exocrine homeostasis, leads to pancreatitis, and facilitates tumorigenesis. Cell Mol Gastroenterol Hepatol 8: 487-511, 2019.

27. Schimmack S, Kneller S, Dadabaeva N, Bergmann F, Taylor A, Hackert T, Werner J and Strobel O: Epithelial to stromal Re-distribution of primary cilia during pancreatic carcinogenesis. PLoS One 11: e0164231, 2016.

28. Kobayashi T, Nakazono K, Tokuda M, Mashima Y, Dynlacht BD and Itoh $\mathrm{H}$ : HDAC2 promotes loss of primary cilia in pancreatic ductal adenocarcinoma. EMBO Rep 18: 334-343, 2017.
29. Li D, Zhu J, Firozi PF, Abbruzzese JL, Evans DB, Cleary K, Friess $\mathrm{H}$ and Sen S: Overexpression of oncogenic STK15/BTAK/Aurora A kinase in human pancreatic cancer. Clin Cancer Res 9: 991-997, 2003.

30. Plotnikova OV, Nikonova AS, Loskutov YV, Kozyulina PY, Pugacheva EN and Golemis EA: Calmodulin activation of Aurora-A kinase (AURKA) is required during ciliary disassembly and in mitosis. Mol Biol Cell 23: 2658-2670, 2012

31. Plotnikova OV, Seo S, Cottle DL, Conduit S, Hakim S, Dyson JM, Mitchell CA and Smyth IM: INPP5E interacts with AURKA, linking phosphoinositide signaling to primary cilium stability. J Cell Sci 128: 364-372, 2015.

32. Garcia-Gonzalo FR, Phua SC, Roberson EC, Garcia G, Abedin M, Schurmans S, Inoue T and Reiter JF: Phosphoinositides regulate ciliary protein trafficking to modulate hedgehog signaling. Dev Cell 34: 400-409, 2015.

33. Kisseleva MV, Wilson MP and Majerus PW: The isolation and characterization of a cDNA encoding phospholipid-specific inositol polyphosphate 5-phosphatase. J Biol Chem 275: 20110-20116, 2000.

34. Bielas SL, Silhavy JL, Brancati F, Kisseleva MV, Al-Gazali L, Sztriha L, Bayoumi RA, Zaki MS, Abdel-Aleem A, Rosti RO, et al: Mutations in INPP5E, encoding inositol polyphosphate-5-phosphatase E, link phosphatidyl inositol signaling to the ciliopathies. Nat Genet 41: 1032-1036, 2009.

35. Kisseleva MV, Cao L and Majerus PW: Phosphoinositide-specific inositol polyphosphate 5-phosphatase IV inhibits Akt/protein kinase B phosphorylation and leads to apoptotic cell death. J Biol Chem 277: 6266-6272, 2002.

36. Phua SC, Chiba S, Suzuki M, Su E, Roberson EC, Pusapati GV, Schurmans S, Setou M, Rohatgi R, Reiter JF, et al: Dynamic remodeling of membrane composition drives cell cycle through primary cilia excision. Cell 168: 264-279.e15, 2017.

37. Mukhopadhyay S, Wen X, Ratti N, Loktev A, Rangell L, Scales SJ and Jackson PK: The ciliary G-protein-coupled receptor Gpr161 negatively regulates the sonic hedgehog pathway via cAMP signaling. Cell 152: 210-223, 2013.

38. Chávez M, Ena S, Van Sande J, de Kerchove d'Exaerde A, Schurmans S and Schiffmann SN: Modulation of ciliary phosphoinositide content regulates trafficking and sonic hedgehog signaling output. Dev Cell 34: 338-350, 2015.

39. Emoto K, Masugi Y, Yamazaki K, Effendi K, Tsujikawa H, Tanabe M, Kitagawa Y and Sakamoto M: Presence of primary cilia in cancer cells correlates with prognosis of pancreatic ductal adenocarcinoma. Hum Pathol 45: 817-825, 2014.

40. Thayer SP, di Magliano MP, Heiser PW, Nielsen CM, Roberts DJ, Lauwers GY, Qi YP, Gysin S, Fernández-del Castillo C, Yajnik V, et al: Hedgehog is an early and late mediator of pancreatic cancer tumorigenesis. Nature 425: 851-856, 2003.

41. Bailey J, Mohr A and Hollingsworth M: Sonic hedgehog paracrine signaling regulates metastasis and lymphangiogenesis in pancreatic cancer. Oncogene 28: 3513-3525, 2009.

42. Deng YZ, Cai Z, Shi S, Jiang H, Shang YR, Ma N, Wang JJ, Guan DX, Chen TW, Rong YF, et al: Cilia loss sensitizes cells to transformation by activating the mevalonate pathway. J Exp Med 215: 177-195, 2018.

43. Bangs FK, Miller P and O'Neill E: Ciliogenesis and Hedgehog signalling are suppressed downstream of KRAS during Acinar-ductal metaplasia in mouse. Dis Model Mech 13: dmm044289, 2020.

44. Baretti M, Ahuja N and Azad NS: Targeting the epigenome of pancreatic cancer for therapy: Challenges and opportunities. Ann Pancreat Cancer 2: 18, 2019. 